\title{
Loose Plant Architecture 1-Interacting Kinesin-like Protein KLP Promotes Rice Resistance to Sheath Blight Disease
}

\author{
Jin $\mathrm{Chu}^{1}$, Han Xu${ }^{1}$, Hai Dong ${ }^{1 *}$ and Yuan Hu Xuan ${ }^{2^{*}}$ (D)
}

\begin{abstract}
Background: Sheath blight disease (ShB) is a destructive disease affecting rice production. Previously, we have reported that Loose Plant Architecture 1 (LPA1) promotes resistance to ShB. However, the mechanisms by which LPA1 confers resistance against this disease have not been extensively investigated. Notably, interactors that regulate LPA-1 activity remain elusive.

Findings: In this study, we identified the interaction of kinesin-like protein (KLP) with LPA1 in the nucleus of rice cells by yeast two-hybrid, bimolecular fluorescent complimentary (BiFC), and co-immunoprecipitation (co-IP) assays. To investigate the role of $K L P$ in promoting resistance to $S h B$, wild-type, $K / p$ mutant, and $K L P$ overexpressor (KLP OX) rice plants were inoculated with Rhizoctonia solani AG1-IA. The results indicated that, compared with the wild-type control, klp mutants were more susceptible while KLP OX plants were less susceptible to ShB. Since LPA1 transcriptionally activates PIN-FORMED 1 a (PIN1a), we examined the expression of 8 related PIN genes. The results showed that only the expression of PIN $1 a$ and PIN3b coincided with KLP expression levels. In addition, a chromatin immunoprecipitation (ChIP) assay showed that KLP bound directly to the promoter region of PIN1a but not of PIN3b. Transient expression assays confirmed that LPA1 and KLP transcriptionally activate PIN1a, and that coexpression of KLP and LPA1 had an additive effect on the activation of PIN1a, suggesting that KLP enhances LPA1 transcriptional activation activity.
\end{abstract}

Conclusions: Taken together, our results show that KLP is a novel LPA1 interactor that promotes resistance of rice to ShB.

Keywords: KLP, Sheath blight disease, Transcription activation, Defense, Rice

\section{Findings}

Rhizoctonia solani ( $R$. solani) is a causative agent of sheath blight disease (ShB) in rice (Oryza sativa) that severely affects rice production in China (Savary et al. 1995). Damage inflicted by ShB occurs during the entire rice cultivating period, and mainly affects the leaves, sheaths, and panicles (Savary et al. 1995). When the disease is severe $\mathrm{ShB}$ reduces the yield by up to 50\% (Savary

\footnotetext{
*Correspondence: Insydh@163.com; xuanyuanhu115@syau.edu.cn 'Institute of Plant Protection, Liaoning Academy of Agricultural Sciences, Shenyang 110161, China

${ }^{2}$ College of Plant Protection, Shenyang Agricultural University, Shenyang 110866, China
}

\section{Springer Open}

et al. 2000). Nowadays, fungicide application is the main approach to control ShB, due to a lack of resistant cultivars and resistance-related genes (Savary et al. 2000). However, the use of pesticides results in severe pollution and increases the cost of cultivation. Therefore, there is an urgent need to identify resistance-related genes and to use those genes to obtain resistant rice cultivars to protect rice from ShB.

Extensive studies have shown that overexpression of chitinase, $\beta$-1,3-glucanase, and polygalacturonase inhibiting protein1 (OsPGIP1) could enhance the resistance of rice to $R$. solani (Shah et al. 2009; Mao et al. 2014; Wang et al. 2015). Inducible expression of OsACS2, an 1- (c) The Author(s). 2021 Open Access This article is licensed under a Creative Commons Attribution 4.0 International License, which permits use, sharing, adaptation, distribution and reproduction in any medium or format, as long as you give appropriate credit to the original author(s) and the source, provide a link to the Creative Commons licence, and indicate if changes were made. The images or other third party material in this article are included in the article's Creative Commons licence, unless indicated otherwise in a credit line to the material. If material is not included in the article's Creative Commons licence and your intended use is not permitted by statutory regulation or exceeds the permitted use, you will need to obtain permission directly from the copyright holder. To view a copy of this licence, visit http://creativecommons.org/licenses/by/4.0/. 
aminocyclopropane-1-carboxylic acid (ACC) synthetase that is a key enzyme in ethylene synthesis, promotes rice resistance to blast and sheath blight (Helliwell et al. 2013). Overexpression of BROAD-SPECTRUM RESIST ANCE2 (BSR2) has been shown to increase rice resistance to $R$. solani (Maeda et al. 2019). Salicylic acidtriggered defense mechanisms play an important role in resistance to $R$. solani (Kouzai et al. 2018). OsARS2, Os2H16, and OsGSTU5 are positive regulators of resistance of rice to ShB (Tiwari et al. 2020; Li et al. 2018), while OsARS2 directly regulates Os2H16 via binding of a GT1 cis-element in the promoter region $(\mathrm{Li}$ et al. 2018). A genome-wide association study identified the Fbox protein ZmFBL41 as a negative regulator of the resistance of maize to banded leaf and sheath blight through its interaction with $\mathrm{ZmCAD}$, a monolignol biosynthesis enzyme. The rice homologous gene $O s C A D 8 b$ plays a similar function in the defense against $\mathrm{ShB}(\mathrm{Li}$ et al. 2019). Our recent work demonstrated that the sugar transporter 11 (SWEET11) negatively regulates the defense of rice against ShB (Gao et al. 2018), while the transcription factor DOF11 activates SWEET14 promoting resistance of rice to $\mathrm{ShB}$ (Kim et al. 2020). This is related to ABI3/VP1-Like 1 (RAVL1) that positively regulates the defense of rice against ShB by modulation of brassinosteroids and ethylene signaling (Yuan et al. 2018). Overexpression of Loose Plant Architecture 1 (LPA1), containing an indeterminate domain (IDD), promoted the defense of rice against $\mathrm{ShB}$ via activation of PIN1a (Sun et al. 2019). Furthermore, IDD13, IDD3, and the G-protein $\gamma$ subunit DEP1 interact with LPA1 to differentially regulate the resistance of rice to ShB (Miao Liu et al. 2020; Sun et al. 2020). However, the mechanism by which LPA1 regulates resistance against ShB remains to be investigated.

To investigate the mechanism by which LPA1 regulates the resistance of rice to $\mathrm{ShB}$, we performed a yeast two-hybrid $(\mathrm{Y} 2 \mathrm{H})$ screen. Among potential LPA1 interactors, we identified a kinesin-like protein (KLP). The $\mathrm{Y} 2 \mathrm{H}$ results indicated that LPA1 interacts with KLP and IDD13 (Fig. 1a). Furthermore, a split-GFP assay was performed in rice protoplasts, confirming that LPA1 interacts with KLP in the nucleus, while no visible signal was detected in the negative control (LPA1-nYFP+cYFP)
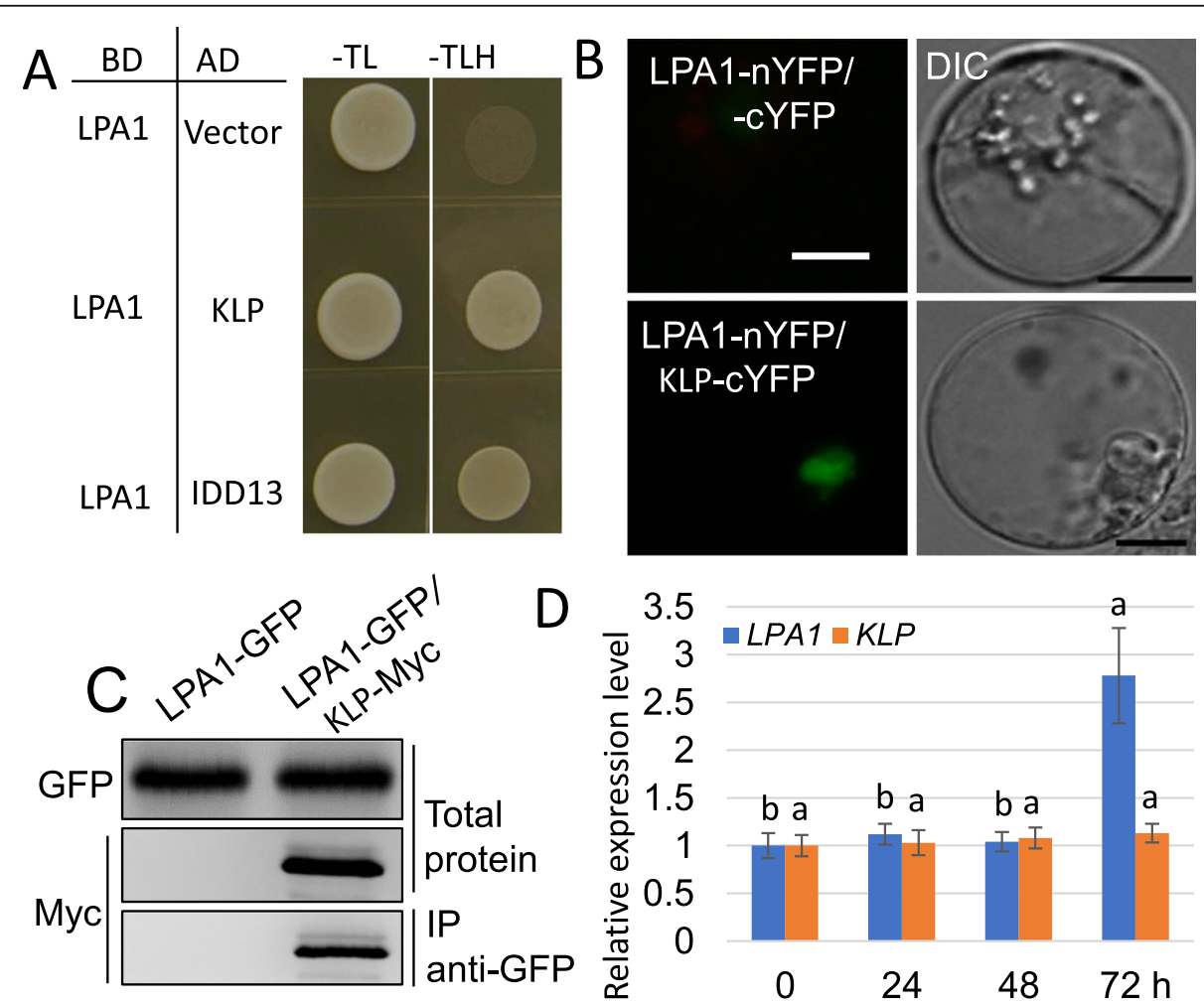

Fig. 1 Interaction between KLP and LPA1. a The interaction between LPA1 and KLP or IDD13 was analyzed by yeast two hybrid (Y2H) assay. BD: GAL4-DNA binding domain; AD: activation domain; -TL: SD medium without tryptophan and leucine; -TLH: SD medium without tryptophan, leucine, and histidine. $\mathbf{b}$ LPA1-nYFP + - cYFP or LPA1-nYFP + KLP-CYFP were coexpressed in rice protoplasts to detect YFP protein reconstruction. Bars $=10 \mu \mathrm{m}$. $\mathbf{c}$ The interaction between LPA1 and KLP was analyzed in tobacco leaves by co-IP. LPA1-GFP+ KLP-Myc or LPA1-GFP were transformed into tobacco leaves using Agrobacterium-mediated transformation. Western blot analysis used an anti-Myc or anti-GFP antibody. Anti-GFP antibody was used to immunoprecipitation. d Relative expression patterns of $L P A 1$ and KLP were examined at $0,24,48$, and $72 \mathrm{~h}$ postinoculation (hpi) with $R$. solani AG1-IA. The error bars indicate the mean $\pm \mathrm{SE}(n=3)$. Different letters indicate significant differences at $P<0.05$ 
(Fig. 1b). In addition, a co-IP was carried out where KLP-Myc was coexpressed with LPA1-GFP in $N$. benthamiana leaves, and an anti-GFP antibody was used to immunoprecipitate LPA1-GFP. Western blot analysis using an anti-Myc or anti-GFP antibody indicated that KLP-Myc and LPA1-GFP were successfully expressed and that LPA1 also interacts with KLP in plants (Fig. 1c). Since $L P A 1$ expression was induced by inoculation of $R$. solani, we also examined KLP expression upon inoculation with $R$. solani. qRT-PCR data showed that LPA1 was induced after $72 \mathrm{~h}$ of the inoculation, but $R$. solani inoculation did not change the expression levels of $K L P$ (Fig. 1d).

To analyze the role of $K L P$ in promoting resistance of rice to $\mathrm{ShB}, k l p$ mutants and $K L P$ overexpression lines were generated. Two independent $k l p$ mutants named $k l p-1$ and $k l p-2$, were generated by insertion of T-DNAs into the 11th intron (Fig. 2a). qRT-PCR data showed that no KLP transcripts were detected in $k l p-1$ and $k l p-2$ mutant plants (Fig. 2b). In parallel, the KLP expression level was examined in wild-type and $4 K L P$ overexpressors $(K L P O X)$ lines $(\# 1, \# 2$, \#3, and \#5). The qRT-PCR data showed that KLP expression levels were higher in KLP1 OXs compared with wild-type plants, and the highest expression was detected in KLP OX \#5 (Fig. 2c). Inoculation with $R$. solani AG1-IA revealed that, compared with wild-type plants, $k l p$ mutants $(k l p-1$ and $k l p$ 2) were more susceptible $(p<0.05)$ while KLP OX plants (\#2 and \#5) were less susceptible $(p<0.05)$ to ShB (Fig. $2 \mathrm{~d})$. The percentage of the leaf area covered with lesions was $39.1 \%$ in WT, $48.2 \%$ in $k l p-1,47.2 \%$ in $k l p-2,27.5 \%$ in $K L P O X \# 2$, and $26.5 \%$ in KLP OX \#5 plants (Fig. 2e).

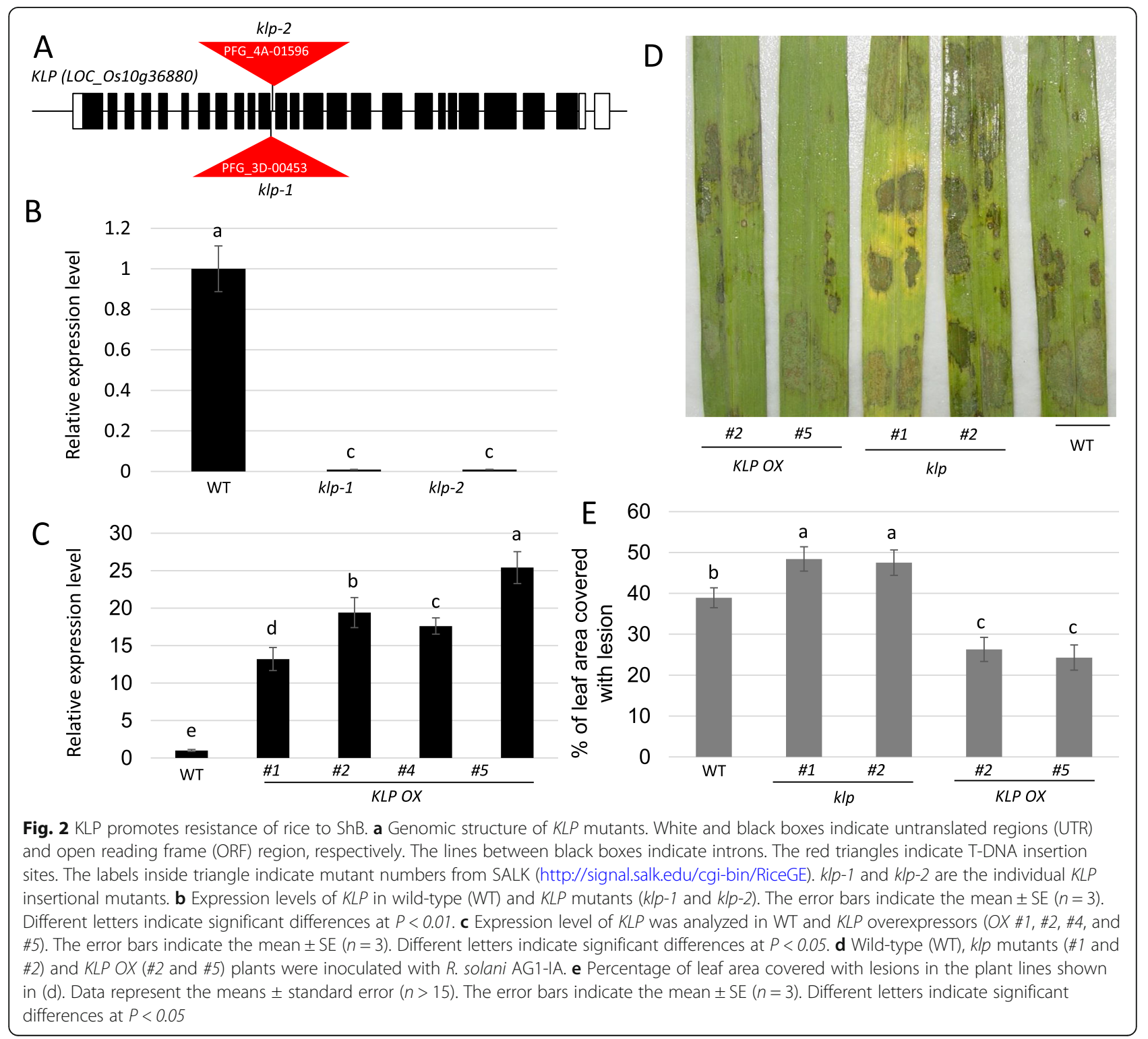


Previously, we have found that LPA1 regulates the resistance of rice to ShB by directly activating PIN1a expression. To test whether KLP also regulates PIN gene expression, the expression levels of 8 PIN genes were analyzed in wild-type, $k l p-1$, and $K L P O X-5$ plants. The results showed that PIN1a and PIN3b expression levels were suppressed in $k l p-1$ and had increased in KLP OX5 plants compared to wild-type plants. PIN1b, PIN1c, and PIN3a expression levels were suppressed in both klp-1 and KLP OX-5 plants compared to wild-type plants. PIN5 $a$ and PIN5b expression levels were higher in KLP OX-5 compared to wild-type plants, while no differences in PIN5a and PIN5b expression levels were observed between wild-type and $k l p-1$ plants. Meanwhile, the expression level of PIN1d was similar between wildtype, $k l p-1$, and $K L P O X-5$ plants (Fig. 3).

Since PIN1a and PIN3b expression was positively regulated by KLP, the affinity of KLP to PIN1a and PIN3b
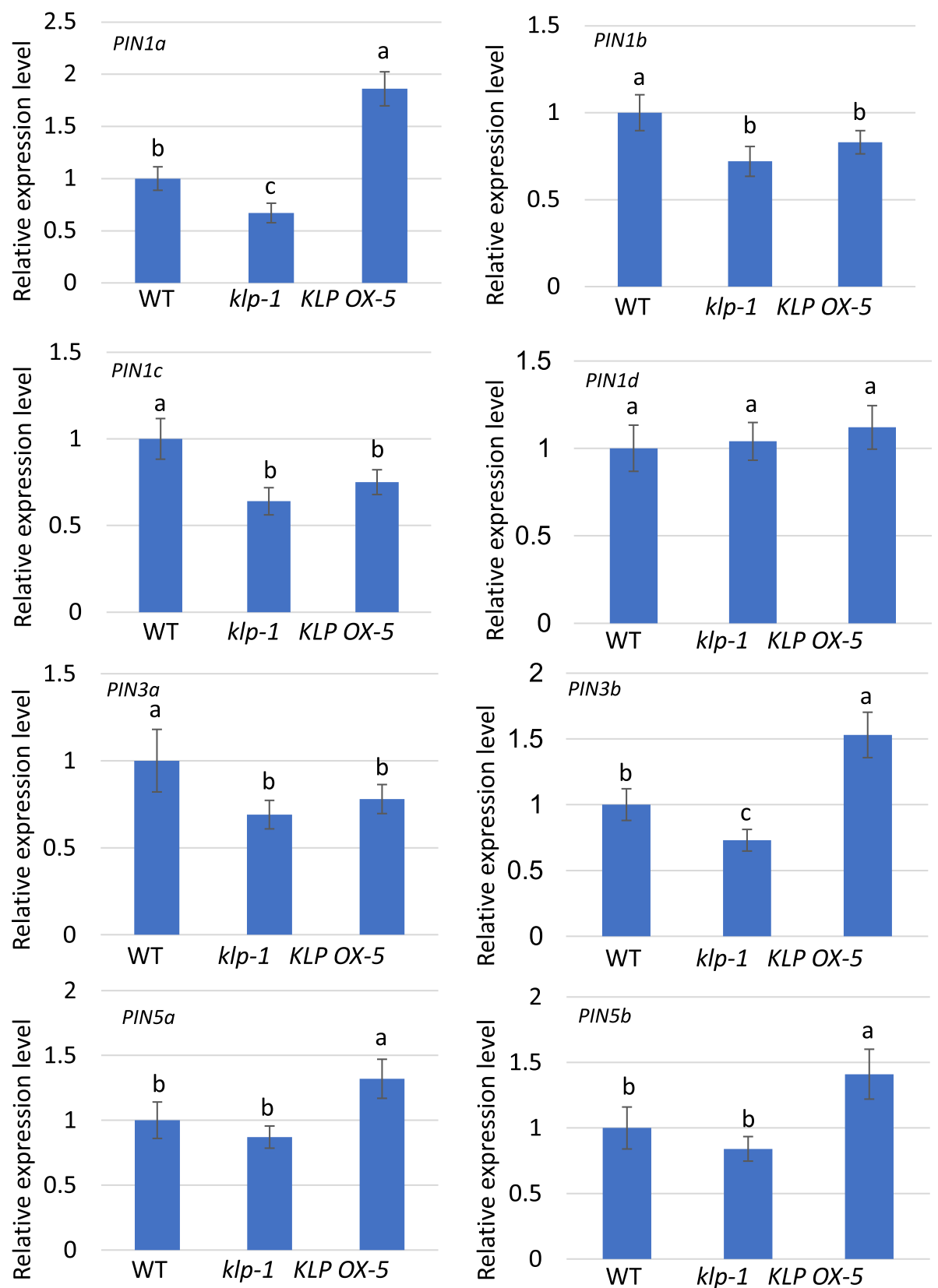

Fig. 3 Expression levels of PIN genes in wild-type, kIp, and KLP OX plants. Shown are the expression levels of PIN1a, PIN1b, PIN1C, PIN1d, PIN3a, PIN3b, PIN5a, and PIN5b in one-month-old, KIp-1 and KLP OX-5 plant leaves relative to WT plants. The error bars indicate the mean \pm SE $(n=3)$. Different letters indicate significant differences at $P<0.05$ 
promoters was examined. Three regions within the 1.5 kb promoter regions of PIN1a (P1-P3) and PIN3b (P4P6), respectively (Fig. 4a), were tested by ChIP PCR using KLP-GFP transgenic plants. The immunoprecipitation was performed using the pre-immune (control) and anti-GFP antiserum. The ChIP-PCR results showed that KLP directly bound to the P3 region of the PIN1a promoter, but no binding affinity was observed in PIN3b promoter region (Fig. 4b). To verify that LPA1 and KLP bind to the P3 region of the PIN1a promoter and activate its expression, transient expression assays were performed using rice protoplasts. The 35S:LPA1, 35S:KLP, or $35 S: L P A 1+35 S: K L P$ plasmids were cotransformed with a construct expressing the $\beta$-glucuronidase gene (GUS) under the control of the $1.5 \mathrm{~kb}$ pPIN1a promoter in the protoplasts. A 35S:Luc (luciferase) plasmid was used as the internal control for evaluation of transformation efficiency (Fig. 4c). Transient assay results showed that LPA1 and KLP activated pPIN1a, and that LPA1 had a higher $p P I N 1 a$ activation activity than KLP. Interestingly, coexpression of LPA1 and KLP resulted in a stronger transcriptional activation of pPIN1a than expression of either LPA1 or KLP alone (Fig. 4d), indicating an additive effect of KLP on LPA1-mediated activation of pPIN1a.

The isolation of resistance-related genes and the breeding of rice plants using these genes is the most efficient way to control disease-mediated loss in rice production. $\mathrm{ShB}$ is a destructive rice disease that causes severe yield reduction. However, the molecular mechanism remains to be determined. Previously, we reported that the IDD-containing protein LPA1 promotes resistance to ShB. In the current study, we have shown that KLP interacts with LPA1 in the nucleus, which was verified by yeast two-hybrid, split-GFP, and co-IP assays. Further genetic analysis using inoculation of KLP mutants and overexpressing plants with of $R$. solani AG1IA strain revealed that KLP promotes rice resistance to ShB. Two independent alleles of $k l p$ mutants were more susceptible while two KLP OXs were more resistant to ShB compared to wild-type plants. These results suggest that KLP is an LPA1-interacting protein that positively

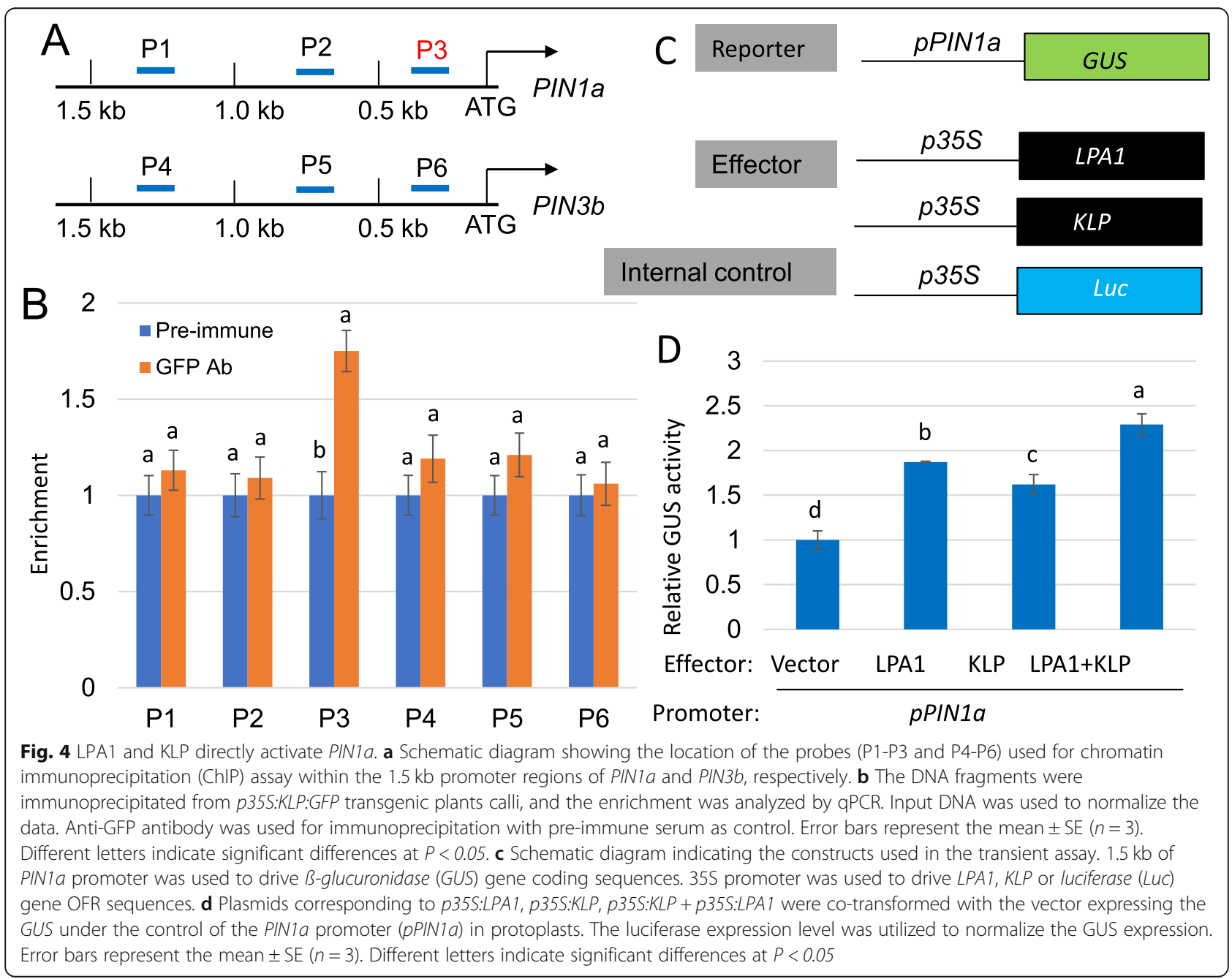


regulates the defense of rice against ShB. Furthermore, our qPCR results demonstrated that PIN1a and PIN3b expression levels positively correlated with KLP levels, while the expression of other PIN genes was differentially regulated by KLP. A ChIP assay using KLP-GFP transgenic plants revealed that KLP directly bound to the PIN1a but not to the PIN3b promoter region. It has been previously reported that the kinesin-like protein BRITTLE CULM12 (BC12) directly binds to the $\mathrm{KO} 2$ promoter of the gibberellic acid (GA) biosynthesis gene directly regulating its expression ( $\mathrm{Li}$ et al. 2011), indicating that a KLP-type protein can function as a transcriptional regulator. Further transient assays confirmed that KLP and LPA1 activate a $1.5 \mathrm{~kb}$ fragment containing the PIN1a promoter, and KLP plays an additive function in LPA1-mediated PIN1a activation. KLP is not transcriptionally activated by infection of $R$. solani, implying that KLP-mediated rice resistance to ShB might be through activation of downstream gene expressions. PIN1a is a polar auxin transporter, and genetic studies have revealed that PIN1a positively regulates the defense mechanism against $\mathrm{ShB}$ in rice. Ethylene functions as positive or negative regulator of plant immunity depends on the type of pathogen, and auxin generally thought of as negative regulator of plant immunity (Yang et al. 2013). Also, ethylene and auxin play opposite role in rice defense to blast disease (Yang et al. 2013), however, exogenous treatment of auxin or activation of ethylene signaling promotes the resistance of rice to ShB (Yuan et al. 2018; Sun et al. 2019), suggesting that auxin and ethylene all play positive role in rice defense to ShB. Also, KLP might regulate PIN1a transcription to modulate local auxin content resulting in increased resistance.

In conclusion, we have shown that KLP, a kinesin-like protein, interacts with transcription factor LPA1 to activate downstream gene expression in a dosage-dependent manner. Our analyses demonstrated that KLP and LPA1 together directly activate PIN1a expression. PIN1a is an ortholog of AtPIN1a, which may control auxin transport to modulate auxin distribution (Petrasek and Friml 2009), and the increase of local auxin concentration promotes resistance of rice to ShB (Sun et al. 2019). Taken together, our results suggest that KLP partners with LPA1, to promote resistance rice to $\mathrm{ShB}$ via activation of PINa-dependent auxin redistribution and subsequent activation of auxin signaling.

\footnotetext{
Abbreviations

KLP: Kinesin-like protein; IDD: Indeterminate domain; ShB: Sheath blight disease; PGIP1: Polygalacturonase inhibiting protein; PIN: PIN-FORMED; LPA1: Loose plant architecture1; SWEET11: Sugar will be eventually exported transporter; RAVL1: Related to ABI3NP1-Like 1; WT: Wild type; OX: Overexpressor; ChIP: Chromatin-immunoprecipitation; Co-IP: Coimmunoprecipitation; BC12: BRITTLE CULM12; GA: Gibberellic acid; GUS: ßglucuronidase; Luc: Luciferase; Y2H: Yeast two-hybrid
}

\section{Acknowledgments}

The authors would thank Dr. Chang-deok Han at Gyeongsang National University, Korea for providing rice seeds.

\section{Authors' contributions}

$\mathrm{J}$ Chu and $\mathrm{YH}$ Xuan designed the experiment and wrote the manuscript. J Chu conducted the experiments and performed data analysis. J Chu and YH Xuan corrected the manuscript. All authors read and approved the final manuscript.

\section{Funding}

This work was supported by the Nature Science Foundation of Liaoning (2020-YQ-05), Nature Science Foundation of China (32072406), and National Key R\&D Program of China (2018YFD02002).

\section{Availability of data and materials}

The datasets supporting the conclusions of this article are provided within the article and its additional files.

\section{Declarations}

Ethics approval and consent to participate

Not applicable.

\section{Consent for publication}

Not applicable.

\section{Competing interests}

The authors declare no conflict of interest.

Received: 15 March 2021 Accepted: 20 June 2021

Published online: 02 July 2021

\section{References}

Gao Y, Zhang C, Han X, Wang ZY, Ma L, Yuan P, Wu JN, Zhu XF, Liu JM, Li DP, Hu YB, Xuan YH (2018) Inhibition of OsSWEET11 function in mesophyll cells improves resistance of rice to sheath blight disease. Mol Plant Pathol 19(9): 2149-2161. https://doi.org/10.1111/mpp.12689

Helliwell EE, Wang Q, Yang Y (2013) Transgenic rice with inducible ethylene production exhibits broad-spectrum disease resistance to the fungal pathogens Magnaporthe oryzae and Rhizoctonia solani. Plant Biotechnol J 11(1):33-42. https://doi.org/10.1111/pbi.12004

Kim P, Xue CY, Song HD, Gao Y, Feng L, Li Y, Xuan YH (2020) Tissue-specific activation of DOF11 promotes rice resistance to sheath blight disease and increases grain weight via activation of SWEET14. Plant Biotechnol J 19:409411

Kouzai Y, Kimura M, Watanabe M, Kusunoki K, Osaka D, Suzuki T, Matsui H, Yamamoto M, Ichinose Y, Toyoda K, Matsuura T, Mori IC, Hirayama T, Minami E, Nishizawa Y, Inoue K, Onda Y, Mochida K, Noutoshi Y (2018) Salicylic aciddependent immunity contributes to resistance against Rhizoctonia solani, a necrotrophic fungal agent of sheath blight, in rice and Brachypodium distachyon. New Phytol 217(2):771-783. https://doi.org/10.1111/nph.14849

Li J, Jiang J, Qian Q, Xu Y, Zhang C, Xiao J, Du C, Luo W, Zou G, Chen M, Huang Y, Feng Y, Cheng Z, Yuan M, Chong K (2011) Mutation of rice BC12/GDD1, which encodes a kinesin-like protein that binds to a GA biosynthesis gene promoter, leads to dwarfism with impaired cell elongation. Plant Cell 23(2): 628-640. https://doi.org/10.1105/tpc.110.081901

Li N, Lin B, Wang H, Li X, Yang F, Ding X, Yan J, Chu Z (2019) Natural variation in ZmFBL41 confers banded leaf and sheath blight resistance in maize. Nat Genet 51:1-9

Li N, Wei S, Chen J, Yang F, Kong L, Chen C, Ding X, Chu Z (2018) OsASR2 regulates the expression of a defence-related gene, Os $2 \mathrm{H} 16$, by targeting the GT-1 cis-element. Plant Biotechnol J 16(3):771-783. https://doi.org/10.1111/ pbi. 12827

Liu JM, Qiong M, XueCY WZY, Li DP, Zhang YX, Xuan YH (2020) Mutation of Gprotein $y$ subunit DEP1 increases planting density and resistance to sheath blight disease in rice. Plant Biotechnol J 19:418-420

Maeda S, Dubouzet JG, Kondou Y, Jikumaru Y, Seo S, Oda K, Matsui M, Hirochika H, Mori M (2019) The rice CYP78A gene BSR2 confers resistance to Rhizoctonia solani and affects seed size and growth in Arabidopsis and rice. Sci Rep 9(1):587. https://doi.org/10.1038/s41598-018-37365-1 
Mao B, Liu X, Hu D, Li D (2014) Co-expression of RCH10 and AGLU1 confers rice resistance to fungal sheath blight Rhizoctonia solani and blast Magnorpathe oryzae and reveals impact on seed germination. World J Microbiol Biotechnol 30(4):1229-1238. https://doi.org/10.1007/s11274-013-1546-3

Petrasek J, Friml J (2009) Auxin transport routes in plant development. Development 136(16):2675-2688. https://doi.org/10.1242/dev.030353

Savary S, Castilla NP, Elazegui FA, McLaren CG, Ynalvez MA, Teng PS (1995) Direct and indirect effects of nitrogen supply and disease source structure on rice sheath blight spread. Phytopathology 85(9):959-965. https://doi.org/10.1094/ Phyto-85-959

Savary S, Willocquet L, Elazegui FA, Castilla NP, Teng PS (2000) Rice Pest constraints in tropical Asia: quantification of yield losses due to Rice pests in a range of production situations. Plant Dis 84(3):357-369. https://doi.org/10.1 094/PDIS.2000.84.3.357

Shah JM, Raghupathy V, Veluthambi K (2009) Enhanced sheath blight resistance in transgenic rice expressing an endochitinase gene from Trichoderma virens. Biotechnol Lett 31(2):239-244. https://doi.org/10.1007/s10529-0089856-5

Sun Q, Li DD, Chu J, Yuan P, Li S, Zhong LJ, Han X, Xuan YH (2020) Indeterminate domain proteins regulate rice defense to sheath blight disease. Rice 13(1):15. https://doi.org/10.1186/s12284-020-0371-1

Sun Q, Li TY, Li DD, Wang ZY, Li S, Li DP, Han X, Liu JM, Xuan YH (2019) Overexpression of loose plant architecture 1 increases planting density and resistance to sheath blight disease via activation of PIN-FORMED 1a in rice. Plant Biotechnol J 17(5):855-857. https://doi.org/10.1111/pbi.13072

Tiwari M, Srivastava S, Singh PC, Mishra AK, Chakrabarty D (2020) Functional characterization of tau class glutathione-S-transferase in rice to provide tolerance against sheath blight disease. 3. Biotech 10:84

Wang R, Lu L, Pan X, Hu Z, Ling F, Yan Y, Liu Y, Lin Y (2015) Functional analysis of OsPGIP1 in rice sheath blight resistance. Plant Mol Biol 87(1-2):181-191. https://doi.org/10.1007/s11103-014-0269-7

Yang DL, Yang Y, He Z (2013) Roles of plant hormones and their interplay in rice immunity. Mol Plant 6(3):675-685. https://doi.org/10.1093/mp/sst056

Yuan P, Zhang C, Wang ZY, Zhu XF, Xuan YH (2018) RAVL1 activates Brassinosteroids and ethylene signaling to modulate response to sheath blight disease in Rice. Phytopathology 108(9):1104-1113. https://doi.org/10.1 094/PHYTO-03-18-0085-R

\section{Publisher's Note}

Springer Nature remains neutral with regard to jurisdictional claims in published maps and institutional affiliations.

\section{Submit your manuscript to a SpringerOpen ${ }^{\circ}$ journal and benefit from:}

- Convenient online submission

- Rigorous peer review

- Open access: articles freely available online

- High visibility within the field

- Retaining the copyright to your article

Submit your next manuscript at $\boldsymbol{\nabla}$ springeropen.com 